\title{
DEVELOPMENT OF INTESTINAL COILING IN ANURAN LARVAE ${ }^{1}$
}

\author{
NORMAN E. KEMP \\ Department of Zoology, University of Michigan, Ann Arbor, Michigan \\ THIRTY-TWO FIGURES
}

In aquatic vertebrates such as the cyclostomes, fishes, and urodele Amphibia, the digestive tract develops as a relatively straight tube characteristically bent toward the right side in the region of the pylorus and duodenum. Anuran amphibians excite particular interest because they are the most primitive vertebrate group to develop a highly coiled intestine, such as amniotes generally possess. Apriori one might think of two extreme alternatives to explain the pattern of the digestive viscera. The first is that these organs are preformed within the endoderm and merely undergo histological differentiation in order to attain their typical position in the fully differentiated larva. The other alternative is that the form of the digestive organs is determined not by the endodermal lining. but by the molding influence of the surrounding mesodermal derivatives.

On the side of the first alternative is the abundant evidence in support of Holtf reter's conclusion that endoderm in Amphibia is regionally determined histologically sometime before gastrulation (Holtfreter, '31, '33, '38a, '38b, '39; Kemp, '46; Stableford, '48), despite claims by Kusche ('29), Balinsky ('38) and Dalcq and Halter ('43) that endoderm retains the capacity for "histological regulation"' (Holtfreter, '38a) subsequent to gastrulation. Presumptive endoderm has also been shown to possess certain intrinsic dynamic tendencies

${ }^{1}$ Aided by a grant from the Horace H. Rackham School of Graduate Studies, University of Michigan. 
prior to the gastrula stage (Holtfreter, '38b, '39, '44; Nicholas, '45; Stableford, '48). Bacon ('45) reports that endoderm of the floor of the archenteron of the early gastrula possesses heart-organizing activity.

Supporting the second alternative is the observation by Holtfreter ('39) that endoderm cultured in salt solution did not form an epithelial tube unless it was in contact with mesoderm. Furthermore, it hàs been repeatedly demonstrated that the digestive tract may undergo "morphological regulation" even as late as the tailbud stage. Born (1896) showed that fusion of overlapping anterior and posterior pieces of two different embryos of Rana esculenta resulted in a larva with a single, continuous digestive tract, despite duplication of parts. In similar experiments on the neurula of Hyla regilla it was shown (Kemp, '46) that complete regulation to normal form might follow duplication of as much as the first half of the midgut. Complete regulation following extirpation of parts of the presumptive digestive tract has been reported by Noka ('30), Holtfreter ('39), Daleq and Halter ('43), and Kemp (op. cit.).

Actually neither of the alternatives stated above affords a complete explanation of the development of the digestive organs. Their morphogenesis should be described rather as a cooperative venture in which early preformation and specific dynamic tendencies within the endoderm are complemented by the modeling influences of extrinsic factors (cf. Holtfreter, '39, p. 181), including: the mesodermal sheath of the gut, the dorsal mesentery, the blood supply, the transverse septum, the osmotic pressure of the fluids in the gut and in the coelom, the available space for expansion, and in the region of the foregut (Stableford, '48) the surrounding tissues derived from the marginal zone. The present paper considers the major mechanical factors, including circulation, hydrostatic pressure, attachments and limited pleuroperitoneal space, which regulate the development of coiling in Anura. 
MATERIALS AND METHODS

Four series of experiments were performed on embryos of Rana pipiens at Shumway stage $17+$, an early tailbud stage (see fig. 5). All embryos were obtained by the usual technique of inducing ovulation with fresh pituitary glands, followed by artificial insemination. Operations were performed in boiled or Seitz-filtered 50\% Holtfreter's solution which had been cooled and shaken to insure adequate dissolved oxygen. Embryos were cultured in the same medium for approximately one day, after which they were transferred to water obtained by running tap water through activated charcoal to remove chlorine, then shaking to restore dissolved oxygen. Animals were observed daily during the progress of differentiation of the viscera and individual specimens dissected and fixed at daily intervals after the second day. Completion of the typical larval pattern of two and one-half intestinal coils (fig. 6) occurred in normal control specimens developing at room temperature approximately 4 days after the stage of operation. At this time the cells of the intestine still contained much yolk. By the 6th post-operative day, however, sections revealed that yolk platelets had entirely disappeared from the intestine. Most of the results recorded in this paper are based on animals cultured 5 to 8 days after the operation. This time is deemed to have been sufficient for maximal visceral morphogenesis even though utilization of endodermal yolk may have been incomplete in some cases.

The operative technique was relatively simple. Parts of the experimental embryos were merely excised either with iridectomy scissors or with the type of microscalpel described by Burch ('42). The exposed surfaces healed rapidly and epidermis generally was able to close in and cover the wound during the first day. At the time of sacrifice animals were immobilized with an electric shock administered through platinum electrodes from a Harvard inductorium. Some specimens in each series were immediately fixed for sectioning. In the majority of specimens, however, the ventral body wall 
was removed with iridectomy scissors or microscalpel in order to expose the viscera prior to fixation in Bouin's fluid. Representative dissected specimens were photographed either on 4- x 5-inch cut film with a $32 \mathrm{~mm}$ Micro Tessar lens or on 35 $\mathrm{mm}$ roll film with a Kine-Exacta camera and extension tubes.

I am indebted to Miss Ruth Gerstner and Mr. Frank Ammermann for their able technical assistance in the microtechnique and photography for this project.

\section{EXPERIMENTAL}

\section{A. Transections of embryos (XS series)}

In the first series of experiments animals were transected along one of the 6 levels depicted in figure 1. Line 1 indicates a plane of transection between the hump of the optic vesicle and the gill plate, separating the anterior part of the head from the rest of the body. Line 2 illustrates an oblique transection passing through the posterior margin of the gill plate, thereby isolating most of the head from a posterior meroplast containing a small amount of presumptive gill arch and presumptive heart. The third line shows a vertical transection just anterior to the pronephros. This was designed to exclude the entire gill plate and presumptive heart from the posterior meroplast. Line 4 transects the embryo just posterior to the pronephros so that approximately the anterior one-quarter of the bodygut is retained in the anterior meroplast. Line 5 cuts through the middle of the bodygut and line 6 separates the anterior three-quarters of the bodygut into the anterior meroplast.

1. XS-1 series. Thirty-nine operations; 23 specimens fixed at least 5 days after operation. Excision of the tip of the head in this series was invariably followed by perfectly normal development of the viscera. Healing was rapid; circulation was established just as in the controls and differentiation proceeded at the same rate. Figure 8 shows a typical specimen dissected 8 days after the operation. The digestive viscera 
cannot be distinguished from those of a normal control. Swimming behavior and reaction to touch were normal in these acerebrate animals (cf. Anagnostis and Rugh, '48). Externally, however, they could be distinguished by their "albino" appearance, lack of eyes, and the truncated shape of the head.

2. XS-2 series. Forty operations; 20 posterior meroplasts fixed at least 5 days after operation. Nine of the 20 specimens considered in this series were successful in completing circulation and hence showed normal differentiation of the digestive tract. Two typical examples are shown in figure 9. Note the crown of gills at the anterior end. Practically the entire

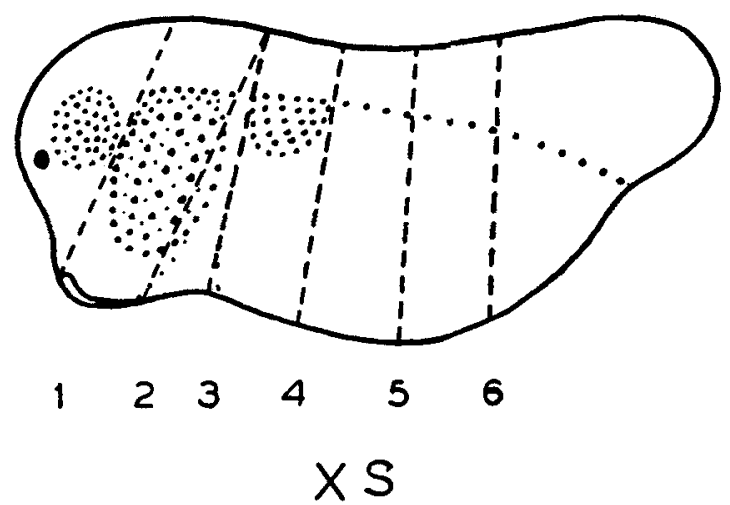

Fig. 1 Diagram of $\mathrm{XS}$ series in which animals were transected along one of 6 different levels illustrated by the broken lines.

head was missing from these specimens; yet sufficient material of the gill plate remained to support a pair of aortic arches and thus permit connection between the ventral and dorsal aortae. The remaining 11 specimens failed to establish circulation even though heart developed and underwent rhythmic contraction. They developed like the two specimens seen in figure 10, in which the digestive tract failed to elongate beyond an advanced S-stage.

3. XS-3 series. Fifty-three operations; 32 fixed at least 5 days after operation. There was virtually no difference in the degree of development of these animals and those of the 
XS-2 series which failed to establish circulation. Figure 11 illustrates two representative specimens. The S-shape of the tract and the retarded differentiation of liver and pancreas, also absence of heart, are obvious features. Other characteristics not clearly brought out in the photograph are: the retention of considerable yolk even though it had all disappeared from control animals; flattening of the tract; and accumulation of fluid in the dorsal mesentery, resulting in balloon-like swelling.

4. XS-4 series. Fifty operations; 15 posterior meroplasts and one anterior meroplast fixed at least 5 days after operation. Embryos in this series were transected just posterior to the pronephros. Approximately the first one-quarter of the midgut was thus isolated in the anterior meroplast. Presumptive liver and pancreas were thus largely excluded from the posterior meroplast. Although anterior meroplasts in this series contained presumptive heart and gills, circulation was not usually established. This probably resulted largely becautse mechanical conditions were unfavorable for completion of a transverse septum, since in the G-2 series animals frequently established circulation even though the amount of bodygut excised was the same as in the present series. Failure of circulation in these animals was accompanied by accumulation of fluid in the coelom. The heart remained nearly straight; stomach and a nearly straight stump of intestine could be distinguished but were relatively undifferentiated. Particularly interesting is the observation that liver segregated asymmetrically, attached to the right side of the incomplete transverse septum. It is evident that establishment of circulation is not prerequisite for the development of asymmetry of the viscera. Posterior meroplasts of this series exhibited S-shaped bending of the intestine without differentiation of liver or pancreas (see fig. 12). Utilization of yolk was obviously retarded in these isolates and the tract was usually flattened.

5. XS-5 series. Fifty operations; 13 anterior and 7 posterior meroplasts fixed at least 5 days after operation. Anterior 
meroplasts in this series received approximately the first one-half of the midgut. Most developed good circulation and showed normal differentiation of the gastroduodenal region. The intestine underwent elongation and became coiled in 8 specimens. One of these had circa one-half coil; 4 had one coil (see fig. 13); one had one and one-half, another two coils (see left specimen, fig. 14). Coiling in all of these was in the normal clockwise direction (viewed from the dorsal side); the 8th specimen (right photograph, fig. 14) had a single coil in reversed or counter-clockwise direction. Since all of these intestines ended blindly within the coelom, it is elear that coiling does not depend upon attachment of the intestine to the body wall posteriorly. It was noted also that the diameter of the intestine was greater than normal in most of the specimens. Evidently excess fluid tended to accumulate and cause swelling. Those anterior meroplasts which failed to establish circulation resembled closely anterior meroplasts of the XS-4 series, exhibiting edema, incomplete transverse septum, and inhibited differentiation of the gastroduodenal region and the stump of intestine. Posterior meroplasts of this series developed a short intestinal loop similar to the posterior loop of the normal S-stage (see fig. 15). There was no evidence of coiling.

6. XS-6 series. Fifty operations; 27 anterior and 4 posterior meroplasts fixed at least 5 days after operation. Approximately three-fourths of the midgut was retained in anterior meroplasts of this series. As in the XS-5 series, the intestine ended blindly within the body cavity and the diameter of the tract was greater than the norm in several specimens. Of 18 animals dissected 4 showed enlargement of the transverse limb of the intestine without coiling; 5 had one coil; 6 had one and one-half coils; one each had one and three-quarters and two coils; one had a single coil in reversed direction. Figures 16 and 17 show 4 of these specimens and illustrate the types of intestinal variation - enlargement, reduced coiling, and reversed direction of coiling. Posterior meroplasts in this series developed only a small, straight intestinal pouch. 


\section{B. Excision of dorsal portions of embryos ( $D$ series)}

The effect of removing portions of the embryo dorsal to the digestive tract was investigated in a series of operations diagrammed in figure 2. In the most drastic operation of this type the entire dorsum - somites, notochord, neural tube and the covering epidermis - was excised. Epidermis soon healed over the isolated ventral portion. Good circulation was established, followed by good differentiation of the digestive viscera. Successively smaller excisions of dorsal anlagen are illustrated by the lines numbered 1 to 6 in the diagram. In
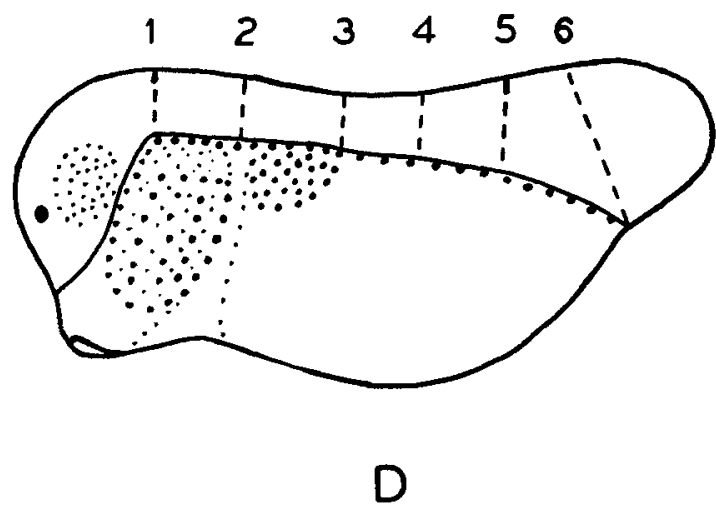

Fig. 2 Diagram of D series, excision of dorsal portions of embryos. Numbered broken lines illustrate levels posterior to which all dorsal material was excised in experiments D-1 to D-6. In the TD series the entire dorsum above the solid line was ablated.

one series all the dorsal material above the yolk border and posterior to line 1 was excised. Similarly, in another series the dorsal tissue posterior to line 2 was ablated, and so on until in series D-6 only the tail bud was removed.

1. D-6 series. Ten operations; 8 fixed at least 5 days after operation. Ablation of the tail bud in this series in no way lessened the normal elongation of notochord and neural tube above the gut. All specimens examined showed perfectly normal eoiling, as, for example, the one pictured in figure 18. At first the operated animals were distinguished by their 
stunted tails notched posteriorly; but regulation was rapid so that by the time of fixation the tails had assumed nearly normal proportions.

2. D-5 series. Ten operations; 7 fixed at least 5 days after operation. Removal of the tail bud and dorsal anlagen above the last one-quarter of the bodygut in this series was followed likewise by normal differentiation of the digestive organs (fig. 19). There did not appear to be any reduction of size of the body cavity in comparison with the norm. A well-developed tail grew out from the plane of excision but not as far as in the previous series.

3. $D$-4 series. Twenty-six operations; 8 fixed at least 5 days after operation. In this series dorsal material as far forward as the middle of the bodygut was excised. Posterior growth of notochord and neural tube was evident; but they did not extend back over the posterior part of the body cavity. A stunted tail did develop from mesenchyme left at the posterior end of the embryo (fig. 20). All specimens showed normal intestinal coiling, proving that it is not necessary for the posterior end of the digestive tract to be surmounted dorsally by notochord and neural tube in order for normal elongation and coiling to take place.

4. D-3 series. Ten operations; 4 fixed at least 5 days after operation. Dorsal material as far forward as the posterior boundary of the pronephric hump was excised in this series. All of the bodygut except the anterior one-quarter was thus freed of overlying notochord and neural tube. Here again normal coiling ensued (fig. 21). A rudimentary tail developed from posterior mesenchyme may be seen in the photograph. As in the D-4 series, this tail was not continuous with the stump of the dorsal organs more anteriorly. This experiment shows that the dorsal organs may be absent over a major part of the intestine and still normal coiling can occur.

5. D-2 series. Ten operations; 6 fixed at least 5 days after operation. The operation in this series removed dorsal tissue as far anteriorly as the posterior border of the gill plate, hence as far forward as the posterior end of the foregut. The 
results differed from those of the previously described dorsal excisions (D-6 to D-3 series). Two of 5 specimens dissected after 6 days of culture showed normal coiling (see fig. 22). The other three animals, however, showed abnormal coiling of the intestine. In all three the stomach lay transversely, crowded toward the anterior part of the body cavity. From the duodenum on the right side the intestine led transversely to the left side, where it continued into a very irregular, twisted portion before ending in the usual straight, terminal portion. The abnormal looping started in a normal clockwise direction in one specimen but in the reverse direction in the other two. The transverse position of the stomach and the abnormal twisting of the intestine in these specimens are interpreted as effects of compression of the coelomic space available for intestinal elongation. Removal of the dorsal organs above the entire midgut, therefore, may result in failure of the body cavity to elongate sufficiently to permit normal coiling.

6. D-1 series. Twenty-five operations; 11 fixed at least 4 days after operation. All of the presumptive dorsal organs as far forward as the anterior border of the gill plate were excised in this series. Seven specimens were dissected. Of these, three exhibited practically normal coiling (e.g., top specimen, fig. 23). Another (bottom specimen, fig. 23) showed anterior displacement of the stomach and but one intestinal coil. The remaining three also showed anterior displacement of the stomach. Coiling was absent in two of these (e.g., middle specimen, fig. 23) ; the other had a beginning coil but in reversed direction. The abnormalities in these animals, like those of the D-2 series, are interpreted as results of compression due to reduced size of the body cavity.

7. TD series. Thirty-seven operations; 28 fixed at least 5 days after operation. The entire dorsum of the embryo was excised in this series; but the embryo retained pharynx, gill plates, and presumptive cardiac anlagen, besides the entire bodygut. 
In the majority of specimens, epidermis healed over the dorsal surface, circulation was established, and good differentiation of the digestive tube proceeded. One group of 10 animals was operated upon in January, 1949, at stage 17, before the tail bud had started to project noticeably. Dorsal healing was excellent and 6 specimens showed good visceral differentiation when fixed 5 to 7 days subsequent to operation. In comparison with controls the bodies of these experimental animals are shorter and the anterior end of the head truncated (see fig. 25). No dorsal organs are present above the body proper; but well-developed tails project posteriorly. Sections revealed that notochord and neural tube were present in these tails. Two specimens are particularly interesting because they developed more than the usual number of intestinal coils, $3 \frac{1}{2}$ and $4 \frac{1}{2}$, respectively. The latter of these animals is depicted in figure 25. It is logical to believe that increased coiling resulted from slight reduction of size of the body cavity in the operated animals. The same growth potential of the digestive tracts in two separate coeloms would carry the intestine relatively farther in the smaller. Two other experimental animals in this group developed with normal coiling - two and three coils respectively. Two others exhibited reduced coiling which may be ascribed to excessive restriction of coelomic space.

Another group of 16 animals was operated upon in November, 1949, at stage $17+$, after the tail bud was well formed. Nine of these healed over well and established good circulation. In contrast to the January group, however, none developed normal coiling. Of 7 animals dissected and fixed 5 days after operation 4 are seen in figure 24. Displacement of the stomach to a transverse position close to the transverse septum is an obvious abnormality. The transverse portion of the intestine beyond the duodenum lay crowded against the stomach in three of these. Another obvious abnormality in this group of animals is reduction of coiling. Five of the 7 exhibited irregular coiling starting in the reversed or counterclockwise direction; one (lower left, fig. 24) showed a single 
coil in normal direction; the remaining animal had merely an S-shaped tract ending blindly in a solid thin strand. The abnormalities encountered in this group are exactly those seen in certain animals of the D-1 and D-2 series. Undoubtedly their explanation is the same: insufficient space for normal elongation.

\section{Excisions of portions of bodygut ( $G$ series)}

In this series of operations ventral portions of embryos were excised by cutting frontally above the "yolk border" and dorso-ventrally along one of the four lines shown in figure 3.

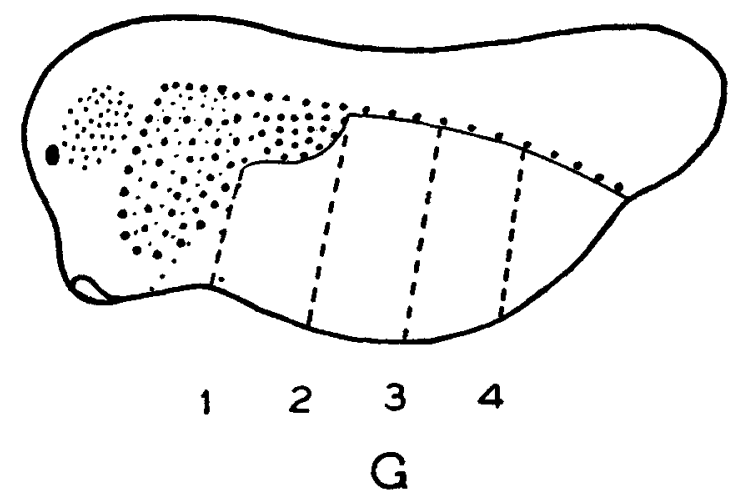

Fig. 3 Tiagram of $G$ series, exeision of ventral portions of embryos, including transverse sections of the bodygut. Broken lines indicate levels posterior to which all ventral material was extirpated in experiments G-1 to G-4.

In experiment G-1 the entire ventral side of the embryo posterior to line 1 , hence including the entire bodygut, was removed. The last three-quarters of the bodygut was removed in experiment G-2, the last one-half and last one-quarter respectively in experiments G-3 and G-4.

1. G-1 series. Twenty-five operations; 9 fixed two days after operation. Care was taken in the operation not to remove the pronephric humps. With this exception, however, the entire ventral side of the body posterior to the region of presumptive heart was excised. Virtually the entire blood island was removed by the operation (see Fernald, 43) and 
removal of the entire bodygut precluded establishment of any vitelline circulation. Two days after the operation only 11 of the 25 experimentals were alive. They resembled anterior meroplasts of the XS-4 series in exhibiting edema, incomplete transverse septum, and relatively straight, pulsating heart. A small rounded mass of undifferentiated endoderm extended into the coelom. Of two animals cultured further, one lived until the 6th post-operative day but died before it could be fixed.

2. G-2 series. Twenty-five operations; 8 fixed at least 5 days after operation. The rate of survival in this series was considerably greater than in the G-1 series. Retention of the first one-quarter of the bodygut and part of the blood island permitted establishment of good circulation; hence two days after operation 15 of the 24 specimens still living showed circulation of blood through the gills. The digestive viscera in these underwent good differentiation at a rate comparable to that of the controls. Of the 8 animals fixed at least 5 days after operation 6 were dissected. They all showed excellent differentiation of the gastroduodenal region and the transverse limb of the intestine (see fig. 26); yet the digestive organs were obviously displaced anteriorly. Three of the 6 specimens showed no coiling (e.g., fig. 26). One, however, had a single coil in normal direction and two had single coils in reversed direction.

3. G-3 series. Forty-one operations; 27 fixed at least 5 days after operation. Percentage of survival and establishment of circulation among these animals was even greater than in the G-2 series. Intestinal elongation was generally greater, for the embryos retained the anterior one-half of the bodygut. Two days after operation all except 4 were healthy with vigorous circulation of blood. Of the 27 later fixed 22 were dissected. Two typical specimens are seen in figure 27. Extent of coiling in this series varied as follows: a transverse, uncoiled intestine -1 specimen; one coil in normal direction -7 specimens; one or slightly more than one coil in reversed direction -13 specimens; two and one-half normal coils -1 
specimen. Most of the animals (e.g., right specimen, fig. 27) exhibited intestinal enlargement. One other specimen besides the apparently normal animal, however, had an intestine of normal diameter. A photograph of this animal (left specimen, fig. 7) clearly shows that the intestine ends in an anus-like fistula. This correlation between normal diameter of intestine and patent terminal opening to the outside cannot be without significance.

4. G-4 series. Thirty-five operations; 20 fixed at least 5 days after operation. All animals were alive two days after the operation. Sixteen of the differentiated specimens were dissected. Eight or $50 \%$ of these showed normal intestinal coiling with two or two and one-half coils of normal diameter. The remainder showed: one enlarged coil in the normal direction - 4 specimens; one enlarged coil in reversed direction - 3 specimens; one coil starting in normal direction but continuing in the reversed direction -1 specimen. In this series, as in the G-3 series, normal diameter of the intestine was correlated with the presence of a patent terminal opening. Figure 28 illustrates the marked contrast between an intestine ending blindly within the body cavity and one opening to the outside. Enlarged diameter and reduced coiling of the former are clearly apparent. This series proves that the intestine is capable of regulation to normal form following excision of the posterior one-quarter of the bodygut.

\section{Excisions from the ventral half of the bodygut (VG series)}

These operations were designed to test the regulatory capacity of the digestive tract following ablation of large sections of the ventral half of the bodygut. The experimental procedure, diagrammed in figure 4, was very similar to that employed in the $G$ series. Embryos were cut frontally along a level half way between the dorsal and ventral sides of the bodygut and vertically so as to eliminate the entire ventral one-half of the bodygut (VG-1 series), the posterior one-half 
of this amount ( $\mathrm{VG}-2$ series), the anterior one-half (VG-3 series), or the central one-half (VG-4 series).

1. VG-1 series. Fifteen operations; 8 animals fixed at least 5 days after operation. Twelve animals were alive with apparently normal circulation 5 days after the operation; 8 were still healthy when fixed on the following day. All showed normal coiling (see fig. 29), although perhaps slightly less than the normal average of two and one-half coils. These

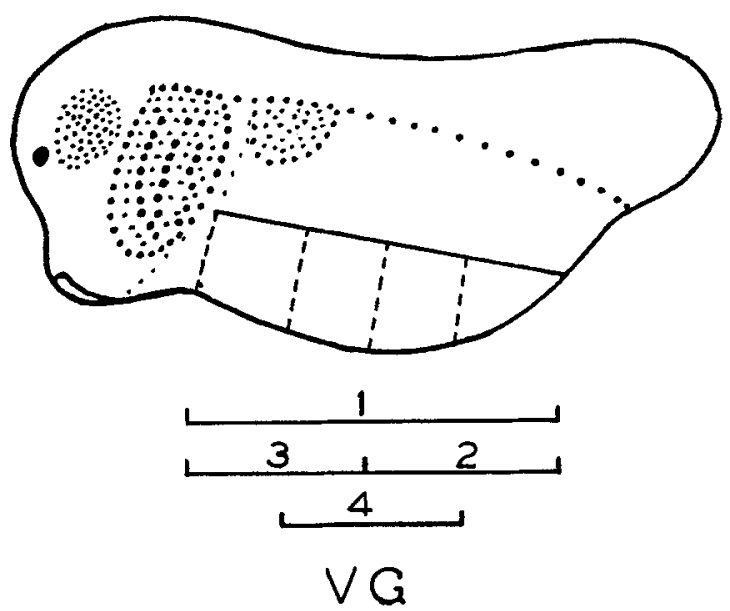

Fig. 4 Diagram of VG series, excision of all or parts of the ventral half of the bodygut. In experiment VG-1 the entire ventral half of the bodygut beneath the solid black line was removed. Excision of the posterior half of this ventral fraction is designated experiment VG-2. Removal of the anterior half is VG-3 and of the central half, VG-4.

results prove that regulation to normal form may follow excision of as much as the entire ventral one-half of the bodygut.

2. VG-2 series. Thirty operations; 19 fixed at least 5 days after operation. A representative specimen dissected on the 6th post-operative day is seen in figure 30. Normal coiling was discovered in all but one of 17 animals dissected. Obviously, normal coiling is to be expected following excision of the posterior one-half of the ventral half of the bodygut.

3. VG-3 series. Thirty operations; 8 fixed at least 5 days after operation. Figure 31 pictures a typical specimen 5 days 
after excision of the anterior half of the ventral half of the bodygut. Coiling was normal in all of 5 dissected specimens.

4. VG-4 series. Thirty operations; 16 fixed at least 5 days after operation. In this series a central section of the ventral half of the bodygut was excised. Normal coiling (see fig. 32) developed in 8 out of 10 dissected specimens; one showed a slight reduction to one and three-quarters coils and the other but one coil located on the right side of the coelom. This one case of situs inversus of the intestine was not accompanied by situs inversus of the gastroduodenal region, hence could not have resulted from primary reversal of the asymmetrical relationship between viscera and transverse septum.

\section{DISCUSSION}

1. Establishment of circulation. The present study has demonstrated that circulation of blood is necessary for completion of differentiation of the digestive viscera. There are at least 4 ways in which this might be brought about. First, the blood stream is a highway for carrying oxygen to the differentiating cells of the gut and for transporting carbon dioxide away. It is possible that increased availability of oxygen is an important stimulus for endodermal histogenesis. In this connection it should be borne in mind, however, that differentiation of certain tissues - epidermis, neural tube, and some mesodermal derivatives - is well advanced even before circulation commences. Second, the circulating blood may transport humoral agents which speed endodermal growth and differentiation. These hypothetical factors aparently do not include hormones from the pituitary gland, since normal digestive organs developed in animals lacking the anterior part of the head (XS-1 and XS-2 series). A third function of the blood stream is the purely mechanical one of dividing the peripheral surface of the endoderm into a number of "islands" enclosed within a network of vessels. Gaseous exchanges and transfers of water, inorganic salts and organic nutrients are undoubtedly facilitated by the close proximity of the surface 
cells to some part of the vascular network. The fourth function of the vascular system is its very important part in helping to maintain the osmotic equilibrium which controls the hydrostatic pressure of the coelom and enteric cavity. Digestive tracts in specimens without circulating blood were usually collapsed (posterior meroplasts of XS-3, XS-4 and XS-5 series), while specimens with circulating blood usually had tubular tracts.

2. Hydrostatic pressure. In the development of a tubular structure such as the intestine it is to be expected that the hydrostatic pressure both in and outside the tube would exert important influence. Fluctuations of pressure within either the enteron or the coelom might cause either deflation or inflation from the "normal"' diameter. These changes in volume would, in turn, be expected to have an important influence on intestinal elongation and coiling. Deflation occurred, as noted above, when the digestive tract lacked circulation. The opposite effect, swelling of the tract, occurred in several series (anterior meroplasts of the XS-5 and XS-6 series, and the G-2, G-3 and G-4 series) in which circulation was established but in which the intestine ended blindly within the coelom. It appears that the open anus normally serves an important function in allowing escape of fluid accumulating in the intestine.

3. Coelomic space. Provision of adequate absorptive surface of the intestinal epithelium is a problem which the vertebrates have met principally by increasing the length of the digestive tract or by internal folding. Comparison of the relative length of the body cavity in larval cyclostomes, fishes and urodele amphibians with that of anuran larvae reveals that all except the latter group have solved their "problem of intestinal length" by developing a long, narrow body cavity paralleling the relatively straight course of the digestive tract. The anuran larva, on the other hand, has a short, broad coelom in which elongation is effected by coiling. If size of the body eavity is a factor in the development of intestinal coiling in Anura, what is the effect of experimentally diminishing or augmenting the coelomic space? 
It is hypothesized that marked compression of the body cavity accounts for the anterior displacement of the viscera and reduced coiling noted in several series of experiments (TD, D-1, D-2, XS-5, XS-6, G-2, G-3, and G-4 series) reported in the present paper. Slight reduction of the body cavity probably accounts for the extra coils in two specimens of the TD series, for if the potential of the intestine for elongation remains the same, the tract should attain greater relative length in a coelom slightly reduced from the norm.

Increasing the size of the coelomic cavity was accomplished in experiments previously described by the author (Kemp, '46). When an anterior portion of one embryo of Hyla regilla at the neurula stage was fused to an overlapping posterior portion of another so that the first two-thirds of the midgut was duplicated, the resulting animal developed an intestine of greatly enlarged diameter, nearly straight for most of its length but terminating in a short, irregularly coiled region. The body cavity in these specimens was noticeably larger than in controls. Reduced coiling probably resulted in part, however, from anchorage of the tract relatively close to the dorsal wall of the coelom at the region of fusion of the two meroplasts. Had the dorsal mesentery been free to increase in height, as in normal development, coiling might also have increased. In another type of experiment, that of excising the body wall at various stages during the development of coiling (Kemp, 49), the intestine has been released completely from the confining space of the normal coelom. One effect of this operation is to permit unwinding of intestine coils already present so that the digestive tract becomes a long, single loop (fig. 7) anchored at its ends by the low dorsal mesentery of the gastroduodenal region and of the terminal portion of the intestine. It is concluded that coiling in Anura results from elongation of the intestine within a body cavity which is neither long nor broad enough to permit development as a straight tube or a single loop. 


\section{SUMMARY}

1. Development of the normal pattern of intestinal coiling: in anurans is dependent upon (1) establishment of the vitelline circulation, (2) regulation of hydrostatic pressure in both the digestive tract and coelom, and (3) the restricted space of the coelomic cavity.

2. Without circulating blood the digestive tract fails to elongate beyond the S-stage of normal development. Utilization of yolk and the cellular rearrangement and mitosis accompanying normal elongation are arrested.

3. Excessive hydrostatic pressure within the intestine leads to swelling and reduced coiling if the gut fails to communicate with the outside through a patent terminal opening. Reduced internal hydrostatic pressure, which results when circulation is not established, causes flattening of the digestive tube.

4. Coiling results from elongation of the intestine within a restricted body cavity. It may occur even though the tract ends blindly within the coelom but is abolished if the body cavity is opened during the development of coiling. Increased coiling may result from slight reduction of the coelom. Excessive coelomic reduction, however, causes crowding of the viscera, reduced amount and abnormal direction of coiling.

5. Morphological regulation to normal form may follow deletions of the entire ventral half of the bodygut, of anterior, posterior or central halves of this fraction, or of the posterior fourth of the bodygut.

\section{LITERATURE CITED}

Anagnostis, I. P., AND R. Rugh 1948 The effect of total brain ablation at stage 28 on subsequent development and behavior in two species of Amblystoma. J. Exp. Zool., 109 : 33-53.

BALINSKY, B. I. 1938 On the determination of entodermal organs in Amphibia. C. R. Acad. Sci., U.R.S.S., Ns 20: 215-217.

BACoN, R. L. 1945 Self-differentiation and induction in the heart of Amblystoma. J. Exp. Zool., 98: 87-125.

BonN, G. 1896 Über Verwachsungsversuche mit Amphibienlarven. Arch. f. Entw.-mech., 4: 349-465, 517-623.

Burch, A. B. 1942 A microsealpel for use in experimental embryology. Science, $96: 387-388$. 
DALCQ, A., AND S. HALTER 1943 Contribution à l'étude expérimentale de la morphogénèse du tractus digestif chez les Amphibiens. Arch de Biol., 54: $477-587$.

Fernald, R. L. 1943 The origin and development of the blood island of Hyla regilla. Univ. Calif. Publ. Zool., 51: 129-147.

HoltFreter, J. 1931 Über die Aufzucht isolierter Teile des Amphibienkeimes. II. Züchtung von Keimen und Keimteilen in Salzlösung. Arch f. Entw.-mech., 124: 404-466.

1933 Die totale Exogastrulation, eine Selbstablösung des Ektoderms vom Fntomesoderm. Entwicklung und funktionelles Verhalten nervenloser Organe. Arch f. Entw.-mech, 199: 669-793.

1938a Differenzierungspotenzen isolierter Teile der Urodelengastrula. Arch f. Entw.-meeh., 138: 522-656.

1938b Differenzierungspotenzen isolierter Teile der Anurengastrula. Arch f. Entw.-mech., 138: 657-738.

1939 Studien zur Ermittlung der Gestaltungsfaktoren in der Organentwicklung der Amphibien. I. Dynamisches Verhalten isolierter Furchungszellen und Entwieklungsmechanik der Entodermorgane. Arch. f. Entw.-mech., 199: 110-190.

1944 Experimental studies on the development of the pronephros. Rev. Canad. de Biol., 3: 220-250.

KFim, N. E. 1946 Regulation in the entoderm of the tree frog Hyla regilla. Univ. Calif. Publ. Zool., 51: 159-183.

1949 Development of intestinal coiling in the anuran tadpole. Anat. Rec., 105: 88.

Kusche, W. 1929 Interplantation umschriebener Zellbezirke aus der Blastula und der Gastrula von Amphibien. I. Versuche an Urodelen. Arch. f. Entw.-mech., 120: 192-271.

Nioforas, J. S. 1945 Blastulation, its role in pregastrular organization in Amblystoma punctatum. J. Exp. Zool., 100: 265-299.

NoKA, H. 1930 Experimentelle Untersuchungen über die Entstehung des Situs viscerum et cordis bei Anurenlarven. Jap. Jour. Med, Sci. Anat., 2: 201-216.

STABLEFORD, L. T. 1948 The poteney of the vegetal hemisphere of the Amblystoma punetatum embryo. J. Exp. Zool., 109: 385-426. 


\section{PLATES}




\section{PLATE 1}

\section{EXPLANATION OF FIGURES}

5 Tailbud embryo of Rana pipiens at Shumway stage $17+$, the stage used for the experiments described in this paper. $\times 8$.

6 Control specimen 7 days after stage of operation. On the right side may be seen the oblique pyloric portion of the stomach with liver anteriorly and panereas posteriorly. Transverse limb of intestine extends from right to left in. the posterior part of the coelom. Approximately two and one-half intestinal coils in elockwise (viewed from the dorsal side) direction. $\times 8 .^{*}$

7 Specinen in which the ventral body wall was excised after complete differentiation of the viscera. Intestine has unwound to form a single loop. $\times 8$.

8 Representative of experiment XS-1, 6 days after excision of tip of head. All digestive organs are normal. Heart may be seen medially between the gills anterior to the level of the transverse septum. $\times 8$.

9 Two representatives of experiment XS-2, 5 days after operation, showing normal differentiation of all digestive viscera after establishment of circulation. Note crown of gills at anterior end. $\times 8$.

10 Two examples of experiment XS-2 in which cireulation was not established, 5 days after operation. The digestive tract has not developed beyond the $\mathrm{S}$-stage. Retarded liver and pancreas are seen anterior to the tract in the right specimen. $\times 8$.

11 Two specimens of experiment XS-3, 5 days after operation. Tract is arrested at the S-stage; undifferentiated liver and panereas at anterior end of tract in right specimen. Dorsal mesentery shows ballooning due to accumulation of fluid. $\times 8$. 


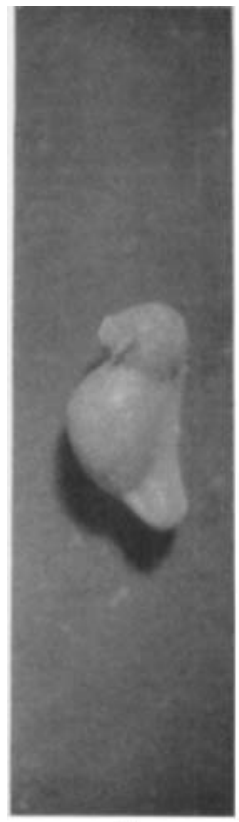

5

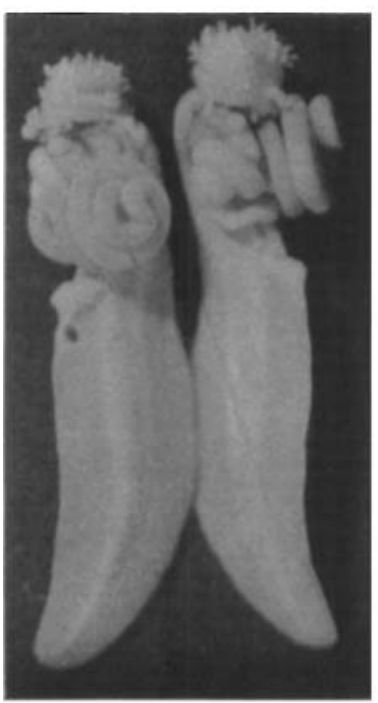

9

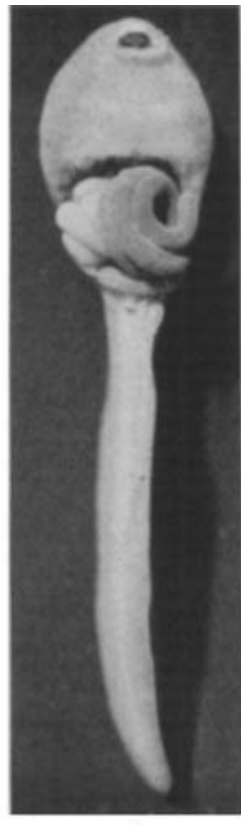

6

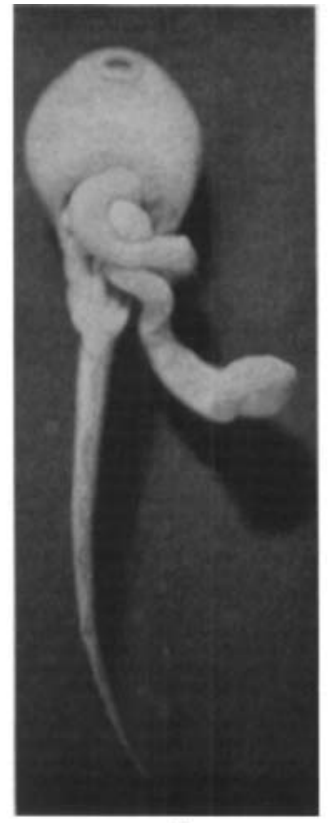

7

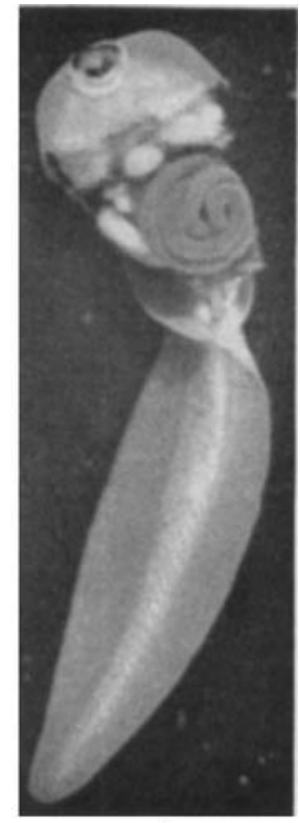

8

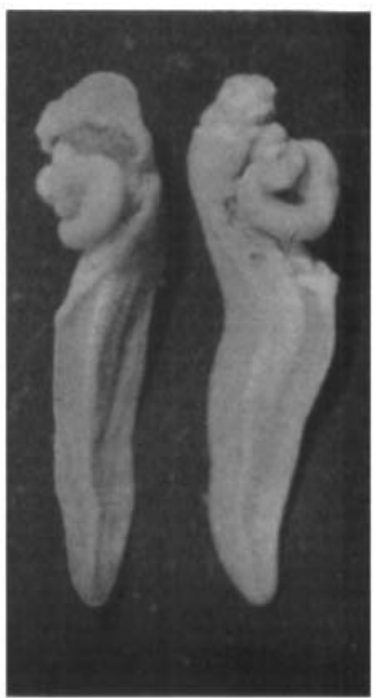

10

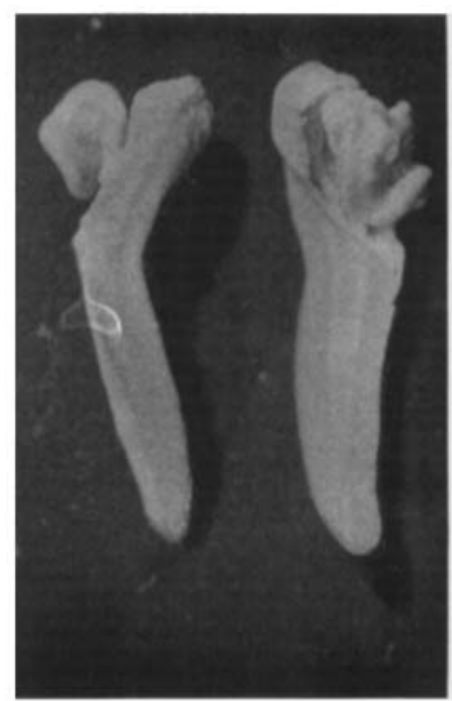

11 


\section{PLATE 2}

\section{EXPLANA'TION OF FTGURES}

12 Two posterior meroplasts of experiment XS-4, 6 days after operation. 'I'ract has developed only to the $\mathbf{S}$-stage and still contains much yolk. $\times 8$.

13 Two specimens of experiment XS-5, 5 days after operation. Approximately one coil has developed in each, although the intestine to the left is collapsed. $\times 8$.

14 Two more examples of experiment XS-5, 5 days after operation. Animal to the left shows two coils in normal direction; animal to right has one coil in reversed (counter-clockwise) direction. $\times 8$.

15 Posterior meroplast of experiment XS-5, 5 days after operation. Intestine has a single loop comparable to the posterior loop of the normal S-stage. $\times 8$.

16 Two examples of experiment XS-6, 5 days after operation. Approximately one and one-half enlarged coils in each. $\times 8$.

17 Two additional examples of experiment XS-6, 5 days after operation. Animal to left has one coil; that to right shows one enlarged coil in reversed direction. $\times 8$. 


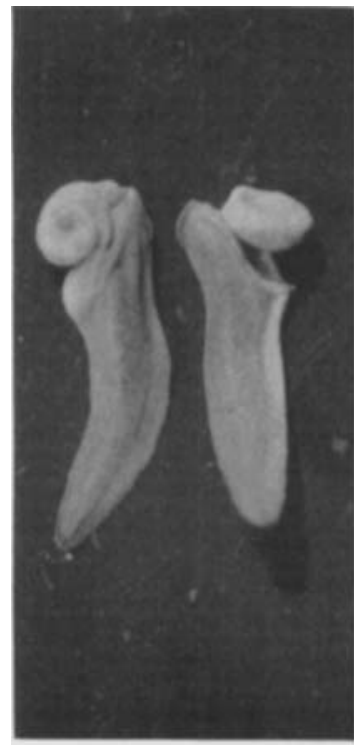

12

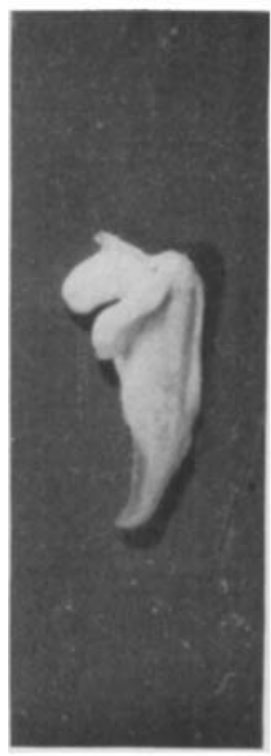

15

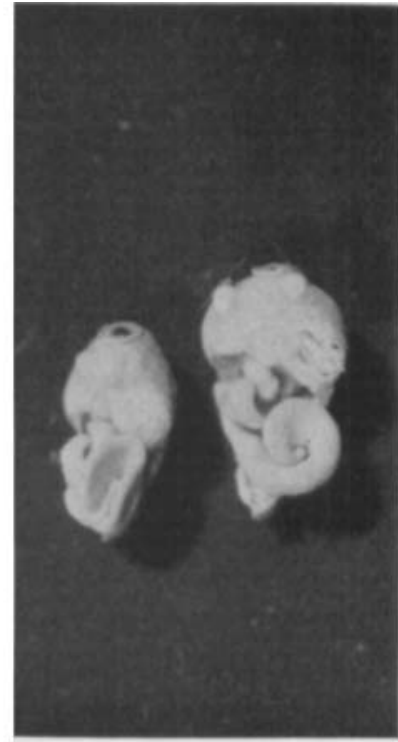

13

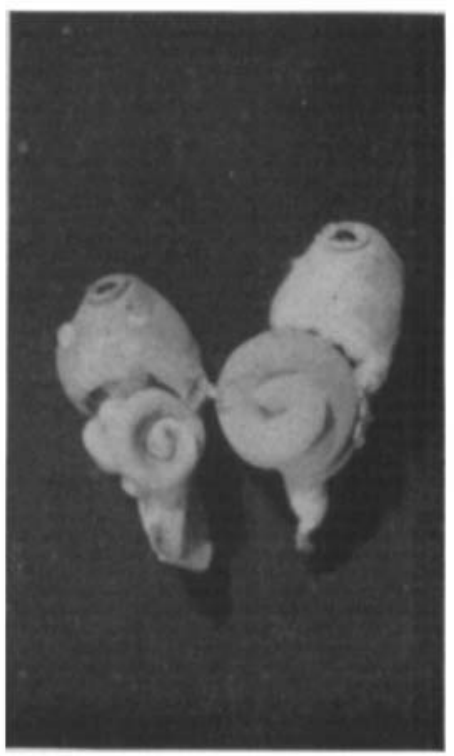

16

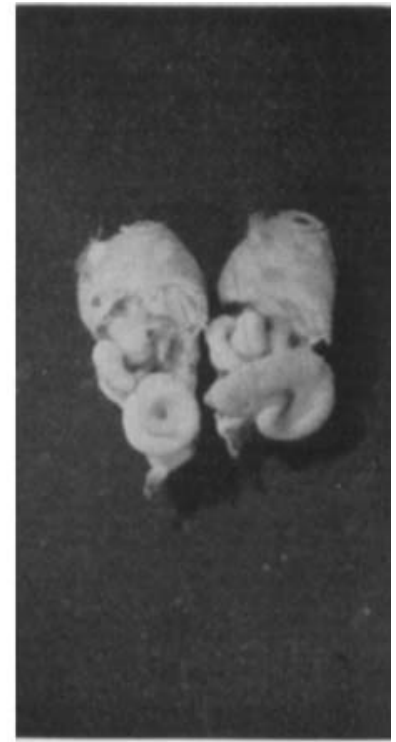

14

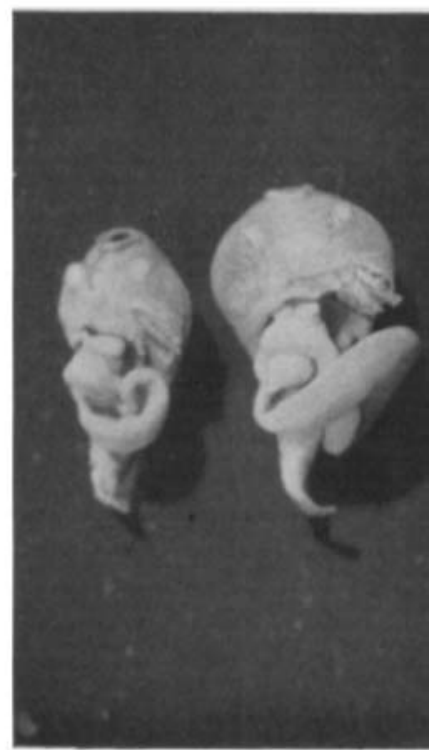

17 


\section{PLATE 3}

EXPLANATION OF FIGURES

18 Representative of experiment D.6, 8 days after operation. Coiling is normal and tail is well-developed. $\times 8$.

19 Example of experiment D-5, 8 days after operation. Coiling is normal but tail is definitely shorter than in D-6. $\times 8$.

20 Example of experiment D-4, 8 days after operation. Coiling is normal. Only a stump of tail is present. $\times 8$.

21 Example of experiment D-3, 8 days after operation. Note normal coiling and stump of tail. $\times 8$.

22 Representative of experiment D-2, 6 days after operation. Normal coiling and stump of tail are seen. $\times 8$.

23 Three specimens illustrating variations in experiment $\mathrm{D}-1,4$ days after operation. Top, normal coiling; middle, uncoiled S-shaped tract; bottom, approximately one and one-half coils but stomach and transverse limb of intestine displaced anteriorly, indicating restriction of size of body cavity. $\times 8$.

24 Four representatives of one group of operations in experiment TD, 5 days after operation. Lower left specimen shows one coil; other three show anterior displacement of stomach and transverse limb of intestine, also irregular coiling in reversed direction. Stump of tail is present. $\times 8$.

25 Representative of another group of operations in experiment TD, 6 days after operation. Operation was performed at Shumway stage 17, which was slightly younger than the stage usually employed. Shows $4 \frac{1}{2}$ coils smaller than normal but in normal position. Tail is well developed. $\times 8$. 


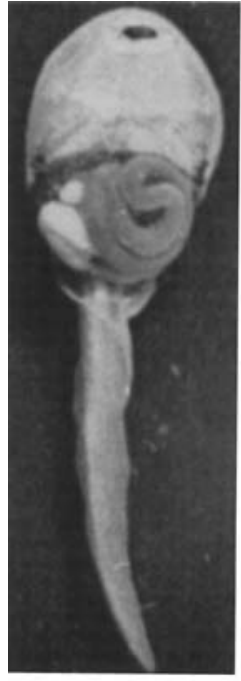

18

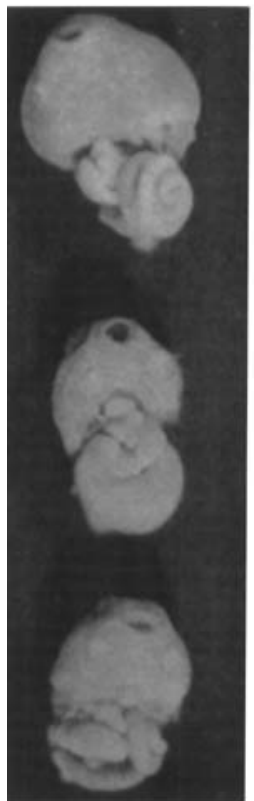

23

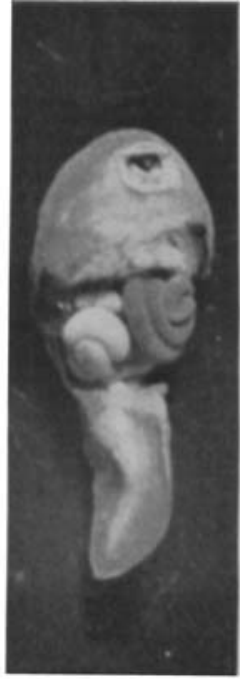

19
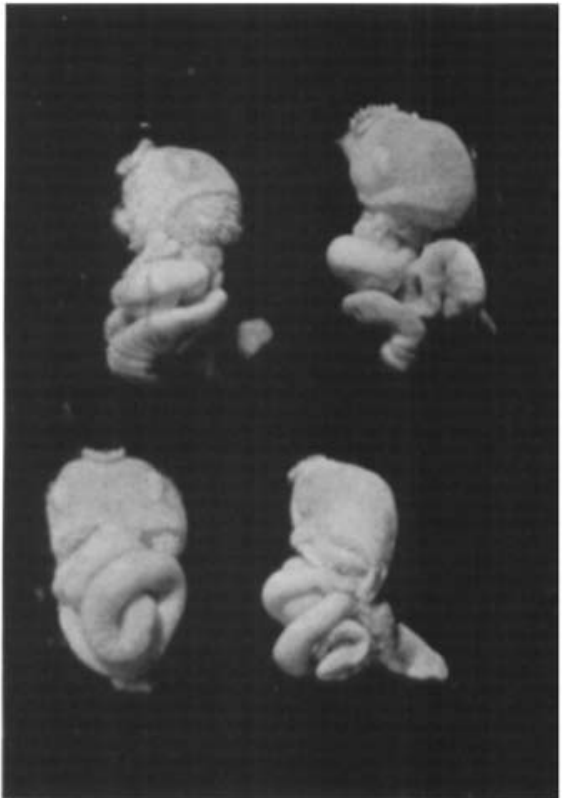

24

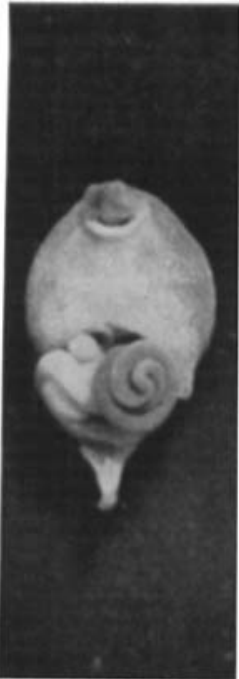

21

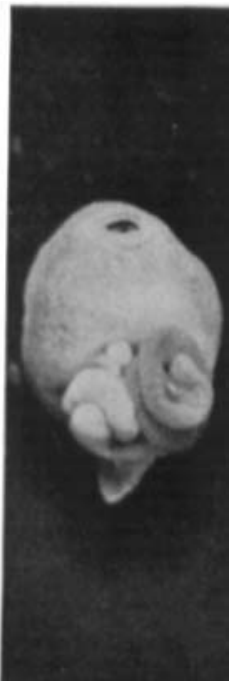

22

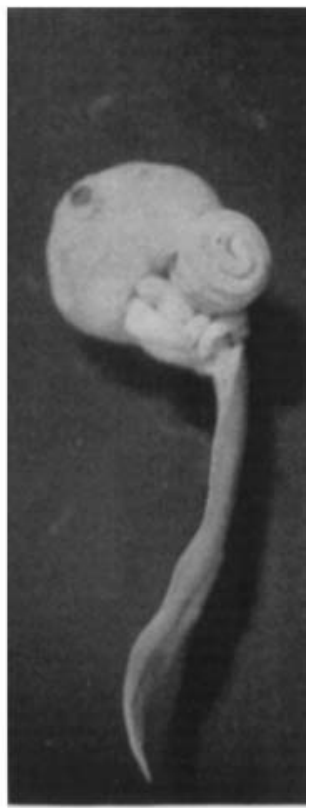

25 
PLA'T' 4

HXPLANATION OH FIGURES

26 Representative of experiment G-2, 6 days after operation. Note enlarged transverse limb of intestine ending blindly within the body cavity. Intestine is uneoiled. $\times 8$.

27 Two examples of experiment G-3, 7 days after operation. Intestine in specimen to left opens to outside through an anus-like fistula and is of smaller diameter than tract to right, which ends blindly within the coelom. $\times 8$.

28 Two examples of experiment G-4, 7 days after operation. These show strikingly enlargement and reduced coiling in the blind tract to the left compared with normal coiling in the intestine to the right, which terminates in an "anal" opening. $\times 8$.

29 Specimen of experiment VG-1, 6 days after operation. Two intestinal coils in normal position. $\times 8$.

30 Specimen of experiment VG-2, 7 days after operation. Two intestinal coils in normal position. $\times 8$.

31 An example of experiment VG-3, 7 days after operation. Shows two and one-half normal coils. $\times 8$.

32 Representative of experiment $\nabla G-4,7$ days after operation. Has two and one-half normal coils. $\times 8$. 


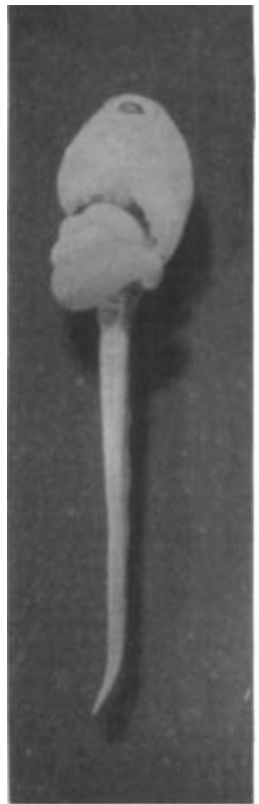

26

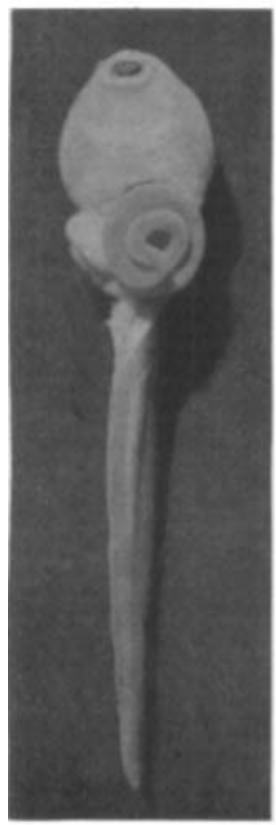

29

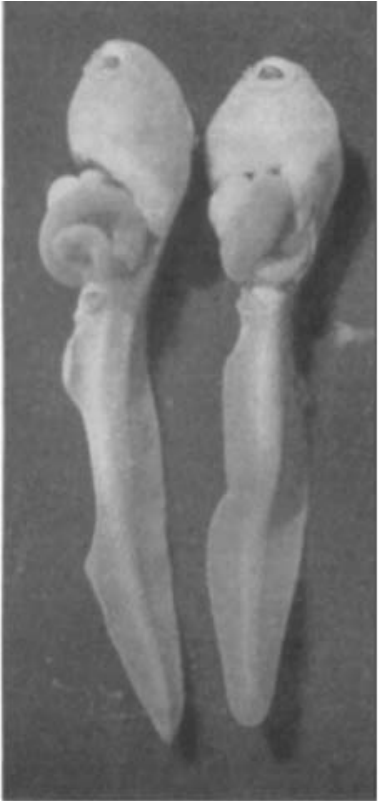

27

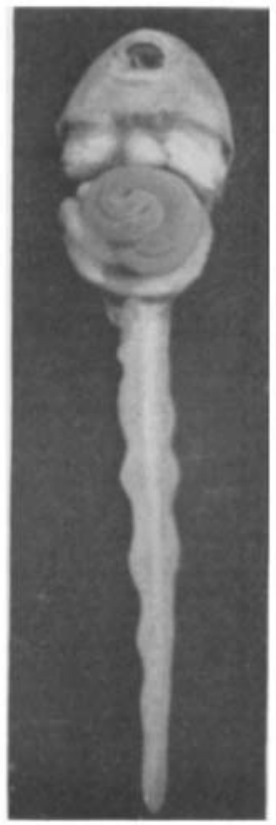

30

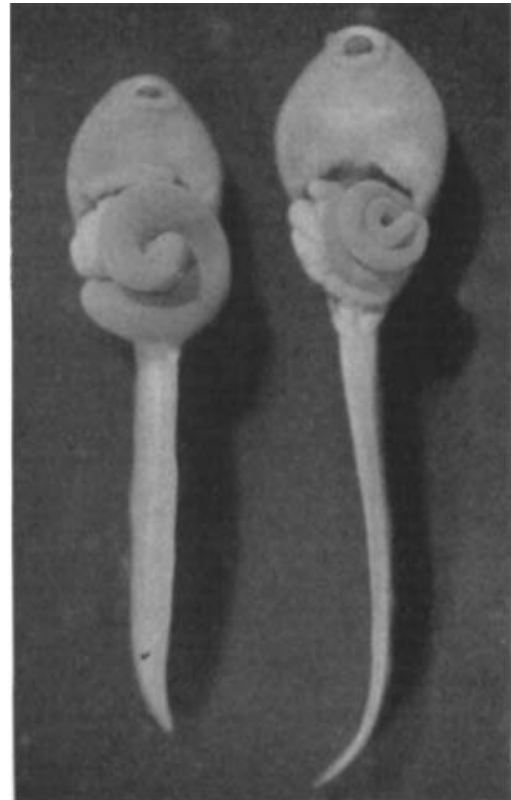

28

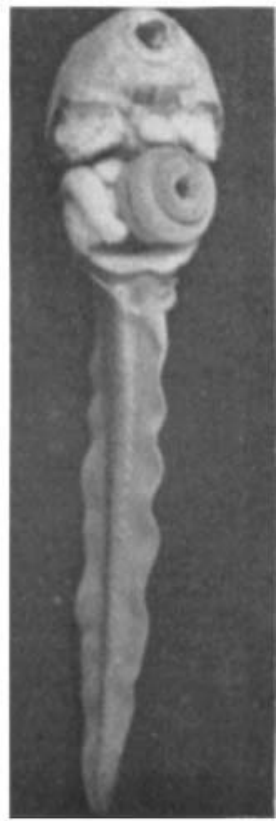

32 